\title{
Comment les jeunes élèves apprennent-ils à jongler avec les occurrences de références en production narrative ? Une étude en temps réel
}

\author{
VICTOR EMMANUEL MILLOGO \\ Université de Poitiers - ESPE de l'académie de Poitiers \\ Laboratoire CeRCA - UMR CNRS 7295
}

\section{Résumé}

Cette étude examine l'impact des occurrences successives d'un personnage sur les paramètres temporels de la production écrite des élèves de 8 à 11 ans. Cinquante-quatre élèves, (16 élèves de CE2 - 8 ans, 18 élèves de CM1 - 9 ans et 20 élèves de CM2 - 10 ans), ont rédigé un texte narratif sur une tablette graphique reliée à un dispositif (Eye and Pen) permettant d'enregistrer leur activité en temps réel. La tâche des élèves était de raconter, sous deux conditions de présentation (deux vs. quatre occurrences du personnage), l'histoire dépeinte par une planche de BD composée de cinq vignettes. Les paramètres temporels (pauses de préécriture, de consultation, d'écriture et temps d'écriture, etc.), correspondant à différentes composantes de l'activité d'écriture, ont été relevés, puis analysés. Les ANOVA ont révélé des différences entre les groupes d'élèves et entre les conditions de présentation. Les analyses corrélationnelles ont montré des fonctionnements différents d'un groupe d'élèves à l'autre au cours de l'activité d'écriture. Ces résultats sont interprétés en termes de développement de stratégie rédactionnelle.

\section{Mots clés}

traitement de la coréférence, occurrences de personnages, chaîne de référence, paramètres temporels, pauses de préécriture, pause d'écriture

\section{Introduction}

L'apprentissage de la production écrite constitue un des enjeux fondamentaux des apprentissages scolaires, au sens où savoir écrire est une compétence essentielle pour la réussite de la scolarisation et pour l'intégration dans la société. Cet apprentissage, qui débute de façon formelle dès les premières années de la fréquentation scolaire ${ }^{1}$ (Ferreiro, 1988), se poursuit tout au long de la scolarité obligatoire, et même au-delà. Sur le plan cognitif, il s'agit d'un apprentissage long et difficile qui implique, de la part du scripteur, de coordonner plusieurs opérations cognitives plus ou moins coûteuses en ressources cognitives, et ce, selon son niveau d'expertise (Piolat, 2004). Depuis les travaux de Hayes et Flower (1980), il est admis que pour produire un texte, le scripteur doit construire au préalable un ensemble de représentations préverbales du message regroupant les idées à communiquer au regard des exigences de la tâche ; ce premier niveau, appelé planification, correspond au niveau sémantique. Ensuite, le contenu du message planifié fait l'objet d'une deuxième opération de traitement pour être mis en mots ; ce deuxième niveau, dit de mise en texte, correspond au niveau linguistique : c'est à ce niveau que

\footnotetext{
${ }^{1}$ Notons tout de même que plusieurs auteurs ont montré que l'enfant se construit une conscience de l'écrit bien avant son entrée à l'école et les progrès futurs en lecture et en écriture dépendent de ces habilités précoces (cf. travaux de Ferreiro, 1988, Gombert et Fayol, 1993, Sénéchal, Lefevre, Smith-Chant et Colton, 2001, Morin et MontésinosGelet, 2007, Fleuret et Montésinos-Gelet, 2011 - pour ne citer que ceux-ci).
} 
s'opéreraient les choix lexicaux, l'organisation syntaxique (textuelle, pragmatique, etc.) et rhétorique du message à produire. Et enfin, les représentations linguistiques construites précédemment sont transformées en réalisation graphique pour être transcrites (niveau graphomoteur); c'est à ce niveau que s'effectue la détermination de l'orthographe correcte des mots, la sélection des allographes, le contrôle de la taille de lettres, l'ajustement musculaire, etc. ; ce dernier niveau de traitement est important puisqu'il consisterait à transcrire les contenus planifiés (van Galen, 1990). Chacun de ces niveaux de traitement fait intervenir une série de processus et de sous-processus fonctionnant en interaction complexe les uns avec les autres, de façon récursive, itérative ou hiérarchique tout au long de l'activité de production, et sollicitant des ressources cognitives. Ainsi, pour le scripteur expert, la rédaction d'un texte consiste à «jongler» constamment avec de multiples opérations cognitives, plus ou moins couteuses en ressources (Fayol, 1986 ; Chanquoy et Alamargot, 2002 ; Olive et Piolat, 2005) ; cela fait dire à Kellogg (1996) que le scripteur est un "penseur" en surcharge cognitive permanente. La complexité des processus en jeu explique, au moins en partie, les difficultés des jeunes scripteurs en production écrite (Hayes et Flower, 1980 ; Berninger et al. 1996). Par exemple, Berninger (1999) estime que chez les jeunes élèves de primaire, la transcription (niveau graphomoteur) rend compte à elle seule environ $66 \%$ de la variance de la fluence de composition (ou aisance d'écriture) et $25 \%$ de la qualité du texte, puis sa part diminue au cours de l'apprentissage pour atteindre respectivement $16 \%$ et $18 \%$ de part d'explication chez les jeunes étudiants. Outre les difficultés liées à la transcription, il est à noter que l'aptitude à coordonner en temps réel les processus de la production de texte dépend, d'une part, de l'efficience des traitements impliqués et, d'autre part, des capacités de la mémoire de travail du scripteur (McCutchen, 1996 ; Olive, 2002).

Les processus en jeu dans la production écrite se développent et se consolident principalement au cours de l'apprentissage scolaire, à travers des exercices de pratiques rédactionnelles variées et répétées, suivant les objectifs d'apprentissage et l'âge des apprenants. Sans prétendre à l'exhaustivité, ces pratiques vont de la simple copie (lettres, mots, phrases et textes), à la rédaction de petits récits (de deux à plusieurs phrases coordonnées), avec ou sans support imagé, en passant par des projets d'écriture multiformes (écrire à la manière d'un auteur, écriture créative, écriture théâtrale, etc.), jusqu'à la production autonome de textes personnels ou fictionnels. Pour atteindre une maîtrise fonctionnelle de la production écrite, les jeunes scripteurs doivent apprendre à gérer de façon coordonnée tous les processus et sous-processus de la production de texte.

Toutefois, les travaux précédemment cités ont porté peu d'attention à la façon dont les jeunes scripteurs (élèves) construisaient leur texte, phrase après phrase, en temps réel. Si la réalisation du texte final constitue l'objectif central de la production écrite, il est tout aussi important d'avoir des indications sur les paramètres temporels de sa réalisation. Ces paramètres temporels sont perçus comme des traces observables de l'activité processuelle et, précisément, comme des indices de charge cognitive (Foulin, 1995). Il a notamment été constaté que l'accroissement de la complexité du thème à traiter s'accompagnait d'une accentuation des pauses (Matsuhashi, 1981). Autrement dit, les variations de certains paramètres temporelles, comme les pauses, renseignent sur la complexité de la mise en œuvre des processus cognitif au cours de l'activité de production (Fayol, Foulin et Chanquoy, 1989). Il apparaît légitime de s'interroger sur l'impact de certaines variables contextuelles du texte à produire (exemple : les occurrences d'apparition d'un personnage) sur les paramètres temporels de la production écrite. En effet, quand il s'agit de produire un texte ou un petit récit, l'activité de production implique de connecter un ou plusieurs segments linguistiques. Ceci implique l'utilisation de plusieurs 
dispositifs linguistiques tels que les marques de ponctuation, les connecteurs et les anaphores pour assurer la cohérence et la cohésion du texte. Le coût des opérations liées au choix et à l'utilisation de ces dispositifs reste encore mal connu. Néanmoins, Fayol et al. (1989) ont montré des distributions différenciées de temps de pauses suivant la nature des marques de ponctuation et de connecteurs. Pour ce qui est des marques coréférentielles, plusieurs recherches concordantes (Karmiloff-Smith, 1985 ; 1992 ; Kail et Hickmann, 1992 ; Hickmann, Kail et Roland, 1995) ont montré que les jeunes élèves éprouvaient des difficultés à les utiliser de façon adéquate dans des situations de narration, du fait d'une maitrise insuffisante de leur fonctionnement. La question qui nous intéresse ici est de comprendre comment, dans le cadre de la production d'un texte de type narratif, les jeunes élèves conçoivent et mettent en œuvre les marques coréférentielles indispensables pour assurer la mention aux occurrences successives d'un personnage.

\section{Définition de la coréférence}

En psycholinguistique, quand deux expressions réfèrent à la même entité sémantique, elles sont dites coréférentielles. La première, généralement désignée sous le terme d'antécédent, introduit l'entité dans le texte et la deuxième, l'anaphore, renvoie à l'antécédent ou première mention du référent ${ }^{2}$ (Ledoux, Gordon, Camblin, \& Swaab, 2007).

Exemple 1 :

a) Jean joue au ballon dans la cour de récréation et [Jean/il/le jeune homme] marque un joli but ;

b) Jean joue au ballon dans la cour de récréation. Jean/il/ le jeune homme marque un joli but.

La coréférence est un mécanisme essentiel pour assurer la cohérence textuelle, car elle permet d'établir des liaisons entre plusieurs phrases du même texte (Grosz, Joshi et Weinstein, 1995). La coréférence peut se réaliser à l'intérieur d'une même phrase comme dans l'exemple 1a, ou à travers deux phrases, comme dans l'exemple $1 \mathrm{~b}$. Elle peut se faire sous plusieurs formes et à travers une variété de marques linguistiques (Corblin, 1995 ; Ariel, 1988 ; 1991): des formes pleines telles que des descriptions définies (déterminant + nom commun), des noms propres, des démonstratifs (déterminant démonstratif + nom commun), des formes vides telles que des ellipses et des pronoms personnels (il ou elle, le, la, etc.) ou démonstratifs (celui, celui-ci, celui-là, celle, celle-ci, celle-là, etc.). Même si chacune de ces marques permet d'assurer la continuité thématique à sa manière, force est de constater que ces différentes marques linguistiques ne véhiculent pas la même connaissance de l'antécédent et de la situation de communication, comme le souligne Ariel (1988). Par exemple, un syntagme nominal tel que «le jeune homme » donne une information sémantiquement riche sur son référent, tandis que le pronom personnel " $i l$ " donne peu d'informations sur son référent. De plus, certaines marques sont plus adaptées que d'autres dans certains contextes. Par conséquent, le choix des marques référentielles en production de texte répondrait à la nécessité de coder l'accessibilité référentielle, c'est-à-dire le niveau de présupposition du référent ou de sa disponibilité en mémoire. C'est ainsi que les pronoms personnels sont plus fréquents quand le contexte linguistique précédent rend l'information référentielle très accessible. C'est ce qui se produit: 1) quand le référent a été récemment mentionné (Ariel, 1990 ; Givon, 1983) ; 2) quand il occupe une position structurale proéminente (position sujet grammatical, position de thème, etc.) dans le segment textuel

\footnotetext{
${ }^{2}$ Dans le cadre de ce travail, nous ne nous traitons pas de la cataphore même si celle-ci constitue également un cas de coréférence
} 
précédent (Arnold, 2001 ; Fukumura et van Gompel, 2010 ; Stevenson, Crawley, \& Kleinman, 1994) ; et 3) quand il n'y a aucun compétiteur référentiel dans le contexte linguistique ou visuel précédent (Ariel, 1990 ; Arnold et Griffin, 2007 ; Clancy, 1980 ; Fukumura, van Gompel, Harley et Pickering, 2010). À l'inverse, les formes pleines (syntagmes nominaux définis, noms propres, etc.) semblent être de meilleurs candidats anaphoriques dans les autres cas de figure (Ferreira, Slevc, et Rogers, 2005 ; Brennan et Clark, 1996). Ce constat empirique ne rend cependant pas compte des mécanismes qui président à la réalisation des marques coréférentielles en production de texte. On peut donc s'interroger sur la façon dont s'opère le traitement de la coréférence au cours de la production de texte, pendant que le scripteur réalise son texte. Cela suppose d'utiliser des techniques adaptées de recueil, ici de temps réel, pour pister les mécanismes en jeu lors du traitement de la coréférence. L'avantage des méthodes de temps réel est de rendre possible l'analyse de l'activité cognitive au cours du déroulement de la tâche. En s'appuyant sur des indicateurs judicieusement choisis, ces méthodes sont susceptibles de décrire le déroulement (décours) temporel des processus - en déterminant à quel moment ils interviennent - et leur agencement avec d'autres processus (Favart \& Olive, 2005).

La présente étude cherche à comprendre comment le traitement de la coréférence (des références successives à un personnage) affecte la production narrative. Plus précisément, dans un contexte où un référent unique est clairement identifié, le nombre d'occurrences du référent affecte-t-il les paramètres temporels de réalisation du texte à produire ? Si le traitement des occurrences successives d'un personnage représente un coût cognitif pour la formulation linguistique, les paramètres temporels (pauses, temps d'écriture, etc.) seront affectés par le nombre d'occurrences coréférentielles, du fait de la nécessité de structurer/organiser la chaîne de coréférence. Autrement, dans des contextes de production comparables, plus les références au personnage seront nombreuses, plus le temps consacré à la structuration/organisation de la chaîne de référence sera important. À l'inverse, si ce traitement n'est pas coûteux en ressources cognitives, le nombre d'occurrences coréférentielles n'aura pas d'effet sur la durée de production. Ces hypothèses sont justifiées par le fait que les marques de référence, comme les chaînes coréférentielles, participent de façon cruciale à l'organisation textuelle. En outre, la constitution des chaînes coréférentielles est soumise à des contraintes d'organisation qui amènent à modifier les formes de reprises à l'intérieur d'une chaîne donnée (Charolles, 1988). Au cours de l'activité, le scripteur doit effectuer un contrôle constant sur les portions de texte déjà écrites et sur les portions à écrire pour établir des interactions entre les différentes formes de reprise (KarmiloffSmith, 1985). Pour tester ces hypothèses, nous proposons à nos participants de produire un récit en s'appuyant sur un support imagé à travers lequel sont manipulés les occurrences d'apparition d'un personnage.

\section{Les facteurs qui affectent le traitement de la coréférence}

Les travaux sur le traitement de la coréférence sont bien plus développés en compréhension qu'en production de texte. Sachant que la plupart des facteurs identifiés en compréhension sont susceptibles d'affecter la production de texte (Ariel, 1988 ; Bates et Devescovi, 1989 ; Fijalkow, 1993), nous prenons appui sur les données actuellement disponibles. Plusieurs travaux attestent que les structures des segments textuels (phrases ou propositions) qui contiennent l'antécédent affectent le traitement des expressions coréférentielles. Les effets de la distance qui sépare l'antécédent et la reprise coréférentielle ont été largement étudiés (O'Brien et al. 1997 ; Gernsbacher, 1989 ; Murphy, 1985 ; Ehrlich \& Rayner, 1983; Brennan, 1995). Il ressort de ces travaux que l'augmentation de la distance entre l'antécédent et sa reprise coréférentielle entraîne une augmentation du temps de traitement de la coréférence. D'autres travaux se sont intéressés à 
la forme de la reprise, syntagme nominal défini versus pronom personnel (Gordon, Grosz \& Gilliom, 1993 ; Camblin et al. 2007), ou au rôle thématique de l'antécédent (agent versus patient; sujet grammatical vs objet grammatical) dans le segment textuel (Albrecht, \& Clifton, 1998 ; Garrod, Freudenthal, et Boyle, 1994 ; Arnold, 2001; Nicol \& Swinney, 1989; O’Brien et al. 1997). Chaque fois, ces travaux ont montré que la structure syntaxique du segment textuel avait un impact sur le décours temporel du traitement de la coréférence. La structure syntaxique du segment de l'antécédent affectait donc l'intégration de l'information référentielle dans la représentation en cours de construction, entraînant un coût de traitement plus ou moins important.

Toutefois, la production de texte présente quelques particularités qui justifient notre intérêt pour cette question. Tout d'abord, l'objectif du traitement est différent: le scripteur choisit d'utiliser une marque référentielle pour assurer la continuité thématique, pour respecter des contraintes de styles (conventions d'écriture par exemple) ou pour éviter les répétitions littérales, alors que le lecteur traite la coréférence pour maintenir son niveau de compréhension du texte. Ensuite, les modalités de traitements diffèrent en mémoire : le scripteur sait à priori ce qu'il a écrit et ce qu'il doit écrire, tandis que le lecteur peut avoir une interprétation plus incertaine de ce qu'il lit. Puis, le scripteur a un rythme d'écriture plus lent que la vitesse de lecture du lecteur : les traitements ne sont donc pas opérés avec la même pression temporelle (Fayol, Foulin et Chanquoy, 1989). Enfin, en production, les ressources cognitives sont en partie consacrées à la réalisation orthographique et graphomotrice du tracé, tandis que ces ressources restent disponibles lors de la compréhension. De plus, le scripteur est le lecteur de son propre texte et la situation de production suppose la prise en compte d'un destinataire médiatisé (Apothéloz, 1989 ; Reichler-Beguelin, 1988). Si le référent est activé en mémoire du fait d'une évocation récente dans le texte déjà produit, alors le scripteur n'a pas besoin de s'y référer explicitement ou alors il choisira des marques référentielles peu accentuées. Du fait de toutes ces particularités, il apparaît pertinent de s'interroger à propos de l'impact des occurrences répétés à un personnage sur la façon dont le scripteur s'appuie sur les marques coréférentielles pour organiser et structurer la progression thématique (Charolles, 1988 ; Reichler-Beguelin, 1988). Corolairement, cela amène à porter une attention particulière à la répartition du coût temporel engagé par le scripteur pour organiser et structurer les marques coréférentielles en production de texte. De plus, il est nécessaire de tenir compte d'autres caractéristiques du scripteur tels que son niveau d'expertise rédactionnelle et sa connaissance du fonctionnement de la langue.

\section{Développement de l'expertise rédactionnelle et du système référentiel}

Les travaux présentés jusqu'ici avaient pour participants des adultes ayant déjà acquis un certain niveau d'expertise rédactionnelle et du fonctionnement de la langue. En revanche, peu de travaux se sont penchés sur la façon dont se mettait en place cette habilité linguistique, ainsi que l'expertise rédactionnelle chez les apprenants. Pourtant, plusieurs études ont montré que l'acquisition du système référentiel était un long processus (Bartlett, 1984 ; Millogo, 2005) nécessitant l'intériorisation d'un certain nombre de règles de fonctionnement, d'un ensemble d'opérations pour intégrer à la fois des aspects sémantiques et pragmatiques des situations de communication (Bates et MacWhinney, 1989 ; Karmiloff-Smith, 1985 ; 1992 ; Kail et Hickmann, 1992 ; Hickmann et al. 1995). Pour l'essentiel, ces travaux s'appuyaient sur les productions narratives, orales et/ou écrites. D'un point de vue méthodologique, les situations narratives apparaissent en effet pertinentes pour l'étude de la coréférence : le scripteur doit non seulement introduire le ou les personnages dans le texte, mais il doit utiliser par la suite des expressions linguistiques spécifiques pour s'y référer sans ambiguïté (Hickmann et al. 1995). Autrement dit, l'utilisation de la production narrative a toujours constitué une activité privilégiée permettant 
d'appréhender la façon dont se mettait progressivement en place le fonctionnement du système référentiel (Botvin et Sutton-Smith, 1977).

En outre, des données concordantes ont permis d'établir un lien étroit entre le développement narratif chez les jeunes enfants et leurs capacités à se représenter les événements du monde (Shiro, 2003, Rathmann, Mann, et Morgan, 2007). Ce développement se traduit par des modifications importantes de structuration et d'organisation du contenu de la narration (cf. McKeough et Genereux, 2003). D'une part, cela se manifeste par une utilisation différenciée des marques référentielles pour marquer le contraste information connue/nouvelle (Vion, Piolat et Colas, 1989) ou pour distinguer le protagoniste principal du protagoniste secondaire (par exemple : savoir que le défini permet d'opposer ce qui est connu à ce qui ne l'est pas, d'assurer la continuité référentielle). D'autre part, il se manifeste par une mise en relation plus efficiente entre les formes et les fonctions (exemple : associer le pronom $i l$ à une fonction de reprise d'un référent déjà connu, de genre masculin et de nombre singulier (Karmiloff-Smith, 1985 ; Hickmann et Schneider, 1993 ; Stadler et Ward, 2005). Les étapes de l'acquisition du système de référence sont donc bien connues chez l'enfant tout-venant, mais cette connaissance s'appuie essentiellement sur l'analyse du contenu des productions, sans prise en compte de l'enchaînement en temps réel des processus qui ont concouru à leur réalisation. Notre étude pallie cet écueil en s'appuyant sur l'analyse d'indicateurs de temps réel pour comprendre comment le nombre d'occurrences d'un personnage dans un contexte de production affecte le temps de réalisation du texte chez des élèves de 8 à 10 ans.

Pour ce qui est du développement de l'expertise rédactionnelle (Bereiter et Scardamalia, 1987 ; Berninger, 1999 ; Berninger, Fuller et Whitaker, 1996 ; Berninger et Swanson, 1994 ; McCutchen, 1996), la question du coût du traitement des expressions référentielles n'a pas été spécifiquement traitée. En revanche, il ressort de ces travaux que les différentes composantes de la production écrite n'évoluent pas au même rythme sur un plan développemental (Berninger et al. 1997) : la transcription est, par exemple, très coûteuse en ressources cognitives en début d'apprentissage, puis elle représente un coût cognitif moindre avec l'automatisation du geste graphique (Berninger et Swanson, 1994). La composante révision serait acquise en dernier (Chanquoy et Alamargot, 2002) : avec le développement de l'expertise rédactionnelle, on assiste à une diversification des retours sur le texte pendant la production en vue de le corriger, à la fois sur sa surface et sur le sens, au niveau des mots, de la phrase puis du texte dans son ensemble (Chanquoy, 2001). D'autre part, ces travaux ont montré qu'en fonction de leur niveau d'expertise rédactionnelle, les scripteurs n'utilisaient pas les mêmes stratégies rédactionnelles (Bereiter et Scardamalia, 1987): les novices (généralement jeunes scripteurs) appliqueraient une stratégie dite de connaissances rapportées, knowledge telling, consistant à transcrire pas à pas les segments textuels à mesure qu'ils étaient planifiés tandis que les scripteurs experts utiliseraient une stratégie dite de connaissances transformées, knowledge transforming. Cette dernière stratégie, plus conceptuelle que la première, permettrait de prendre de la distance par rapport aux contenus préalablement planifiés afin de mieux les adapter aux buts communicatifs. Compte tenu du fait que l'expertise rédactionnelle s'acquiert après plusieurs années de pratique, ces stratégies rédactionnelles ne feront pas spécifiquement l'objet de notre analyse. Toutefois, il est acquis qu'avec l'âge certaines composantes s'automatisent, comme par exemple la transcription, libérant ainsi des ressources pour les autres processus. Il est par conséquent légitime de s'interroger sur la façon dont évolue la coordination des différents processus en fonction de l'âge.

Pour mieux appréhender la dynamique de la production de texte, notre étude se centre sur l'analyse de l'activité en temps réel des scripteurs, en examinant de façon assez précise les paramètres temporels de la production. Cette approche permet ainsi d'évaluer l'impact du nombre 
d'occurrences d'un personnage sur les processus rédactionnels sous-jacents à la production de texte.

\section{Objectif de notre étude}

Notre recherche étudie le coût du traitement de la coréférence lorsque les scripteurs structurent des chaînes coréférentielles en production de texte. Le premier objectif est d'apporter un éclairage sur le coût des traitements liés à la structuration des chaînes coréférentielles en production écrite. Nous voulons savoir comment la longueur de la chaîne de coréférences à organiser et à structurer affecte les paramètres temporels de la réalisation du texte. In fine, l'organisation et la structuration des chaînes coréférentielles modifient-elles les paramètres temporels de la production de texte ? Si oui, comment cela se traduit-il au cours de la production du texte ? Pour le savoir, nous allons examiner une série d'indicateurs de temps de réel recueillis au moment où les scripteurs produisent leur texte. La tâche de production s'appuie sur une planche de bande dessinée dans laquelle les occurrences d'apparition d'un personnage varient sous deux conditions (cf. ci-dessous). Le deuxième objectif est de préciser les modifications qui interviennent dans la production de texte en fonction de l'âge des élèves. Nous voulons savoir comment les différents indicateurs de la production (pauses, temps d'écriture, etc.) interagissent au cours de l'apprentissage scolaire. Y a-t-il des liens entre les différents indicateurs temporels ? Si oui, ces liens sont-ils de même nature quelle que soit la longueur de la chaîne coréférentielle à organiser et à structurer? Sont-ils de même nature d'un groupe d'âge à l'autre ou sont-ils différents ? En effet, les travaux cités ci-dessus, notamment Berninger et al. (1997) ont montré que l'agencement des processus rédactionnels évoluait avec l'expertise.

\section{Participants}

\section{Méthode}

Cinquante-quatre élèves ont été recrutés dans une école primaire publique de la circonscription de Poitiers (Vienne) pour participer à cette étude. Les élèves sont répartis dans trois groupes en fonction de leur classe (niveau scolaire) : 16 élèves de CE2 (G1 : âge moyen = 8;1 ans, écart-type =0,35), 18 élèves de CM1 (G2 : âge moyen = 9;1 ans, écart-type $=0,40)$ et 20 élèves de CM2 (G3 : âge moyen $=10 ; 3$ ans ; écart-type $=0,47$ ). Le niveau de connaissance orthographique des élèves a été évalué avec le test TNO (Test de niveau d'orthographe Doutriaux et Lepez, 1980), édité par les Éditions du Centre de Psychologie Appliquée (ECPA). Ce test a été utilisé comme un outil pour évaluer les connaissances scolaires en langue française (Bourdin, Leuwers et Bourdon, 2011 ; voir également Bouchard, Fitzpatrick et Olds, 2009 pour une discussion). Nous avons utilisé la version du test qui concerne les niveaux de classes situés entre le CE2 et la $5^{\mathrm{e}}$ ( $2^{\mathrm{ème}}$ année du collège dans le système d'enseignement en France). Les résultats de cette épreuve ont attesté que les performances en orthographe grammaticale et d'usage des élèves progressaient en fonction de l'âge : les plus âgés étaient plus performants que les plus jeunes. En outre, au sein d'un même groupe d'âge, les performances des élèves étaient plutôt proches les unes des autres (faibles écarts entre les plus performants et les moins performants).

\section{Matériel et tâches}

Le matériel était constitué d'une planche de bande dessinée tirée d'un album de "Cédric" (Laudec et Cauvin, 1997), dont les séquences ont été modifiées et adaptées pour nos manipulations expérimentales (cf. Annexe $\mathrm{n}^{\circ} 1$ ). Cette planche présentait l'histoire d'un petit garçon (Cédric) à qui il arrive une série d'aventures. Sur cette planche apparait un seul 
personnage (Cédric) dès la première vignette. Puis suivant l'une ou l'autre condition expérimentale, il réapparaissait soit deux fois (condition 1), soit quatre fois (condition 2). Dans tous les cas, l'ensemble de la planche était composé de cinq vignettes. Dans la condition 1, le personnage réapparait dans les vignettes consécutives ( 2 reprises coréférentielles potentielles) à la première apparition du personnage, puis les deux autres vignettes présentaient des éléments du contexte. Dans la condition 2, le personnage réapparaissait dans les quatre vignettes consécutives (4 reprises coréférentielles potentielles).

Les participants voyaient en une seule fois l'ensemble de la planche et celle-ci apparaissait sur un écran d'ordinateur portable disposé en face d'eux (cf. Figure 1). Les participants devaient produire un texte narratif relatant l'histoire de la BD sur une feuille de papier de taille A5 placée sur une tablette graphique connectée à l'ordinateur portable. L'ensemble du dispositif était piloté par le logiciel Eye and Pen (Alamargot, Chesnet, Dansac et Ros, 2006). Ce logiciel enregistrait en temps réel tout ce que le participant réalisait sur sa feuille au cours de la production de son texte : la trace écrite et tous les paramètres temporels.

\section{Procédure}

Avant de commencer l'expérience, les participants étaient familiarisés avec le fonctionnement du dispositif. Ce dispositif comprenait : 1) une tablette graphique dont la surface était partagée en trois zones de fonction (zone d'écriture, zone de consultation et zone de fin), un stylo à encre noire (Inkpen) et une feuille de papier A5 placée sur la zone dédiée de la tablette, et 2) un ordinateur portable Dell qui permettait à la fois d'afficher à l'écran les vignettes et de répondre aux commandes de la tablette graphique (cf. Figure 1). L'ordinateur utilisait le logiciel le logiciel Eye and Pen (Alamargot, Chesnet, Dansac et Ros, 2006) pour piloter l'ensemble du dispositif expérimental et pour enregistrer l'activité d'écriture des participants en temps réel.

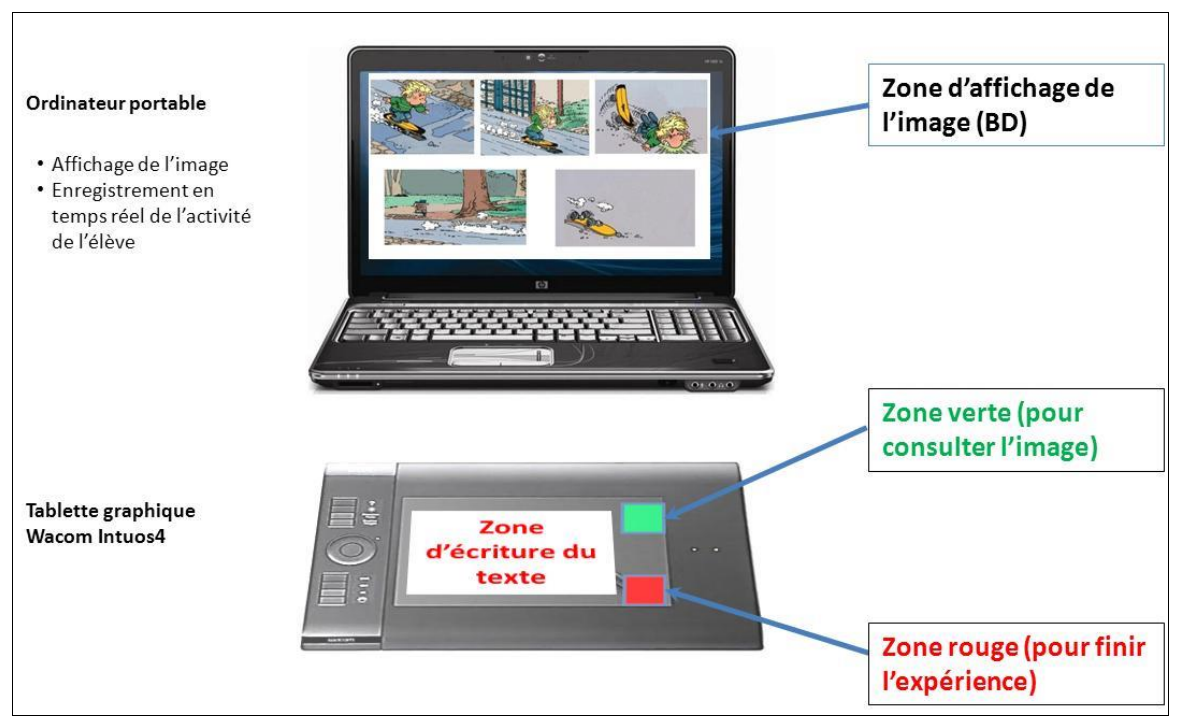

Figure 1 : Dispositif expérimental

Ensuite, l'expérimentateur lisait la consigne suivante à haute voix à chaque participant:

"Vous allez voir apparaitre sur l'écran d'ordinateur une planche de BD. Nous vous demandons de raconter l'histoire de cette bande dessinée à l'écrit à quelqu'un qui ne la connait pas et qui ne la voit pas. Vous devez faire référence à toutes les vignettes sans 
faire une description vignette par vignette. Nous vous demandons d'écrire l'histoire de la BD sur la feuille de papier qui est placée sur la tablette. Quand vous serez prêt, vous pouvez commencer à écrire et la BD disparaîtra. Si vous voulez revoir la BD pour poursuivre votre histoire, vous devez appuyer sur la zone verte et garder le stylo dessus autant de temps que vous le souhaitez. Attention, vous devez raconter l'histoire et non commenter chaque vignette individuelle. Vous ne devez pas vous arrêter quand vous commencez à écrire."

Cette consigne était répétée et reformulée au besoin jusqu'à ce qu'elle soit comprise par les participants.

Enregistrement des données. Le dispositif expérimental permettait de recueillir deux grandes catégories de données : (1) les traces écrites, recueillies sur les feuilles A5, pour l'analyse du contenu des récits produits par les élèves et (2) les données de temps réel pour l'analyse des paramètres temporels de la production. Nous ne présenterons ici que les données de temps réel.

A partir des données de temps réel recueillies avec Eye and Pen (Alamargot et al. 2006), nous avons déterminé cinq mesures temporelles pour constituer nos indicateurs. La première, la durée de la pause de préécriture, correspond au temps qui s'écoule entre l'examen visuel du stimulus et le début de la production du premier mot du texte (Foulin et Fayol, 1988 ; Espéret, Chesnet, Ploquin et Crété, 1995). Selon Dedeyan, Largy et Negro (2006), la pause de préécriture représente le temps de planification initiale de la production écrite. Nous avons déterminé la durée de la pause de préécriture conformément à la définition des écrits du domaine ; ici, il correspond au temps qui s'écoule entre l'affichage initial de la planche de BD et le début de la production du premier signe sur la tablette. La deuxième, la durée totale de production du texte, correspond au temps mis par le participant pour produire son texte. La troisième mesure, le nombre et la durée de consultation de la planche de $\mathrm{BD}$, correspond à la séquence où le participant affiche volontairement sur son écran le stimulus pour le consulter afin de poursuivre sa rédaction. Nous avons relevé de façon exhaustive l'ensemble de ces consultations et leur durée. La quatrième, c'est la durée d'écriture avec le stylo appuyé sur la feuille (transcription graphique). Cette durée d'écriture correspond aux périodes au cours desquelles les participants produisent leur texte de façon continue, sans faire de pause supérieure à $100 \mathrm{~ms}$. La cinquième donnée, la durée des pauses en cours d'écriture, correspond aux périodes au cours desquelles les participants arrêtent d'écrire leur texte, sans pour autant consulter le stimulus (le stylo est immobile plus de $100 \mathrm{~ms}$ ) ; ces pauses, souvent accompagnées ou précédées d'un ralentissement du débit de production, ont été considérées dans les écrits du domaine comme des indicateurs de traitements parallèles ou de retour sur le texte déjà produit (Chesnet et Alamargot, 2005 ; Alamargot, Dansac, Chesnet et Fayol, 2007).

Pour tenir compte des différences interindividuelles et des différences intragroupes, nous avons retenu les indicateurs suivants, correspondant à des processus essentiels de l'activité de production écrite : 1) la proportion de la pause de préécriture (durée de la pause de pré-écriture divisée par la durée totale de production), 2) la proportion de temps consacrée à la consultation (durée des consultations divisée par la durée totale), 3) la proportion des pauses d'écriture (durée des pauses d'écriture divisée par la durée totale de production) ; 4) la proportion de temps consacrée à l'écriture (durée d'écriture divisé par la durée totale de production); 5) le débit de production (nombre de mots produits divisé par le temps total de production); et 6) le nombre de pauses de consultation.

Analyse des données : Nous avons appliqué à nos données deux types d'analyse : 1) une analyse de variance (ANOVA) pour mettre en évidence les différences de fonctionnement entre 
les trois groupes d'élèves en fonction des manipulations expérimentales et 2) une analyse corrélationnelle pour chaque groupe d'élèves pour révéler les liens différenciés entre les différents paramètres de l'activité d'écriture.

\section{Résultats}

Dans un premier temps, nous avons regardé comment la durée totale de production du texte était distribuée en fonction des groupes d'élèves et des conditions expérimentales (cf. Figure 2). Globalement, les durées de pré-écriture varient entre 10,86\% (pour les élèves du CM1) et 21,66\% (pour les élèves du CE2). La durée des pauses de consultations fluctue entre 21,26\% (pour les élèves du CE2) et 46,48\% (pour les élèves du CM1). La durée des pauses d'écriture représente entre 20,47\% (pour le CM1) et 27,12 (pour le CM2). La durée d'écriture (transcription graphique) est comprise entre 19,12\% (pour le CM1) et 32,60\% (pour le CE2). Ces résultats indiquent que le temps consacré à la production du texte n'est pas distribué de la même manière suivant les groupes d'élèves et suivant les conditions ( 2 vs 4 reprises coréférentielles potentielles). Les analyses de variance ci-dessous permettent de mieux apprécier ces différences pour chaque indicateur.

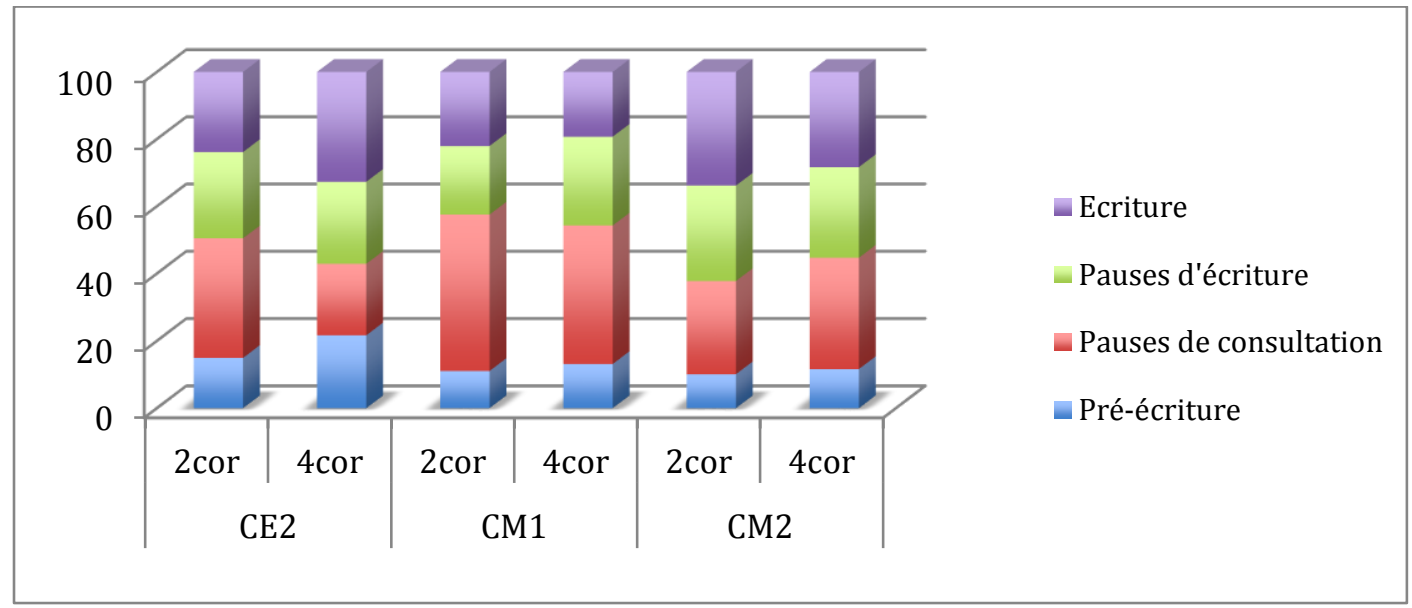

Figure 2: Proportion de temps consacré aux différents comportements au cours de la production en fonction du groupe et des conditions expérimentales

\section{Les analyses de variances}

Dans cette section, nous reportons les analyses de variances 3 (Groupes : CE2, CM1 et CM2) x 2 (Corefs : 2corefs vs. 4corefs). Nous avons associé, pour chaque ANOVA, la valeur du seuil $p$ de significativité et la taille de l'effet êta carré (voir Cohen, 1988 ; Murphy et Myors, 2004 ; Richardson, 2011). Nous présentons ci-dessous les analyses pour chaque indicateur.

La proportion de la pause de préécriture: la proportion moyenne des pauses de préécriture étaient de 10,89\% pour le CM2, 12,09\% pour le CM1 et $18.31 \%$ pour le CE2. Les analyses révèlent cependant une absence d'effet principal du groupe, mais une tendance importante, $\mathrm{F}(2,53)=2,55, \mathrm{p}=0,06, \eta^{2}=0,11$, une absence d'effet de la condition, $\mathrm{F}(1,53)=$ $1,64, \mathrm{p}>0,20$ et une absence d'interaction entre le groupe et la condition, $\mathrm{F}(2,53)=0,38, \mathrm{p}>$ 0,50. Autrement dit, les groupes de participants ne se différenciaient pas significativement par rapport à la proportion de temps de pause consacrée à la préécriture, et ce quelles que soient les conditions de présentation. 
La proportion des pauses de consultation : effet principal du groupe, $\mathrm{F}(2,53)=9,55, \mathrm{p}<$ $0,01, \mathrm{\eta}^{2}=0,29$, indiquant que les participants du CE2 (28,39\%) consacraient moins de temps à la consultation de la planche de BD que les participants du CM2 (30,53\%) et du CM1 (43,82\%); pas d'effet principal de la condition, $\mathrm{F}(1,53)=2,27, \mathrm{p}=0,14$, mais en revanche, un effet d'interaction, $\mathrm{F}(2,53)=3,27, \mathrm{p}<0,05, \mathrm{\eta}^{2}=0,12$. Les comparaisons partielles indiquent des différences entre les deux conditions pour les élèves du CE2 $(\mathrm{p}<0,05)$, mais pas d'effets pour les deux autres groupes.

La proportion des pauses en cours d'écriture : effet principal du groupe (CM1 < CE2 < $\mathrm{CM} 2), \mathrm{F}(2,53)=9,62, \mathrm{p}<0,01, \mathrm{\eta}^{2}=0,29$; effet principal de la condition (2corefs $>4$ corefs), $\mathrm{F}(1,53)=4,49, \mathrm{p}<0,05 ; \mathrm{\eta}^{2}=0,09$ mais pas d'effet d'interaction, $\mathrm{F}(2,53)=1,82, \mathrm{p}=0,17$.

La proportion du temps d'écriture (transcription graphique) : les analyses suggèrent un effet principal du groupe, $\mathrm{F}(2,53)=6,91, \mathrm{p}<0,01, \mathrm{\eta}^{2}=0,23$, indiquant que les participants $\mathrm{du}$ CM1 $(44,08 \%)$ consacrent relativement moins de temps à l'activité d'écriture que les participants du CE2 $(53,30 \%)$ et du CM2 $(58,58 \%)$; mais pas d'effet de la condition $(\mathrm{F}<1)$ et pas d'effet d'interaction, $\mathrm{F}(2,53)=1,68, \mathrm{p}=0,20$.

Le débit de production : effet principal du groupe, $\mathrm{F}(2,53)=14,37, \mathrm{p}<0,01, \mathrm{\eta}^{2}=0,38$, le débit de production est d'autant plus important que les participants sont âgés; pas d'effet de la condition, $\mathrm{F}(1,53)=2,53, \mathrm{p}>0,10$ et pas d'interaction entre le groupe et la condition, $\mathrm{F}(2,53)=$ $1,78, \mathrm{p}>0,10$.

Le nombre de pauses de consultations: effet principal du groupe, $\mathrm{CE} 2=5,94<\mathrm{CM} 2=$ $7,63<\mathrm{CM} 1=9,78 ; \mathrm{F}(2,53)=5,91, \mathrm{p}<0,05, \eta^{2}=0,20$, suggérant que les participants du CE2 consultaient en moyenne moins souvent la planche de BD que les participants du CM2 et du CM1 ; pas d'effet de la condition $(\mathrm{F}(2,53)=0,54, \mathrm{p}>0,50)$ et pas d'effet d'interaction $(\mathrm{F}(2,53)=$ $0,41, \mathrm{p}>0,50)$.

\section{Les analyses corrélationnelles}

Dans cette section, nous présentons les résultats des coefficients de corrélation linéaire $r$ de Bravais-Pearson. Etant donné les faibles effectifs dans chaque groupe, nous avons réalisé un test de normalité (le test de Shapiro-Wilk) sur chaque indicateur et par groupe avant d'appliquer cette méthode. Les résultats de ces analyses préliminaires indiquaient que les distributions de nos indicateurs respectaient globalement les conditions d'application du calcul des corrélations (cf. Howell, 1998). Chaque valeur du coefficient de corrélation est associée à un seuil de significativité (valeur p) correspondant au risque de se tromper en rejetant l'hypothèse statistique d'une absence de liaison entre les deux indicateurs.

Dans un premier temps, des corrélations ont été calculées séparément pour chaque condition de passation, puis dans un deuxième temps, pour chacun des trois groupes d'élèves. Tableau 2).

Les résultats des analyses corrélationnelles sont résumés ci-dessous (cf. Tableau 1 et 
Tableau 1

Corrélations entre les indicateurs de temps réel pour chaque condition

\begin{tabular}{lcccccc}
\hline Condition 1 (2corefs) & 1. & 2. & 3. & 4. & 5. & 6. \\
\hline 1. Nb consultations & 1 & & & & & \\
2. Pause Préécriture & $-0,46^{*}$ & 1 & & & & \\
3. Durée Consultation & $0,56^{* *}$ & $-0,16$ & 1 & & & \\
4. Débit Production & $-0,03$ & $-0,07$ & $-0,30$ & 1 & & \\
5. Pause Écriture & $-0,28$ & $-0,21$ & $-0,54^{* *}$ & $-0,26$ & 1 & \\
6. Durée Écriture & $-0,08$ & $-0,35$ & $-0,58^{* *}$ & $0,62^{* *}$ & $-0,08$ & 1 \\
\hline Condition 2 (4corefs) & & & & & & \\
\hline 1. Nb consultations & 1 & & & & & \\
2. Pause de Préécriture & $-0,45^{*}$ & 1 & & & & \\
3. Durée Consultation & $0,78^{* *}$ & $-0,52$ & 1 & & & \\
4. Débit Production & $-0,18$ & $-0,01$ & $-0,23$ & 1 & & \\
5. Pause Écriture & $-0,27$ & $-0,45^{*}$ & $-0,14$ & $-0,29$ & 1 & \\
6. Durée Écriture & $-0,29$ & $-0,02$ & $-0,61^{* *}$ & $0,56^{* *}$ & $-0,21$ & 1 \\
\hline
\end{tabular}

Note : corrélation significative à $: * \mathrm{p}<.05 ; * * \mathrm{p}<.01$

Condition 1 ( 2 corefs). Les analyses révèlent cinq corrélations significatives. Le nombre de consultations est négativement corrélé à la proportion de temps consacrée à la préécriture $(\mathrm{r}=$ $-0,46, \mathrm{p}<0,05)$, indiquant que plus les participants consacraient du temps à la préécriture, moins ils consultent la planche de BD par la suite. Le nombre de consultations est positivement corrélé à la proportion de temps consacrée à la consultation $(\mathrm{r}=0,56, \mathrm{p}<0,001)$ indiquant que plus les participants consultaient la planche de BD, plus ils y consacraient du temps. La durée de consultation est négativement corrélée à la proportion de temps consacrée à la pause en cours d'écriture $(\mathrm{r}=-0,54, \mathrm{p}<0,05)$ : plus la durée de consultation est importante, moins les participants consacraient du temps à la pause en cours d'écriture. La durée de consultation est négativement corrélée avec la proportion de temps consacrée à l'écriture $(r=-0,58, p<0,01)$. Le débit de production est positivement corrélé avec la proportion de temps consacrée à l'écriture ( $\mathrm{r}$ $=0,62, \mathrm{p}<0,01)$.

Condition 2 (4 corefs). Les analyses de données indiquent cinq corrélations significatives. Le nombre de consultations est négativement corrélé avec la proportion de temps consacrée à la préécriture $(\mathrm{r}=-0,45, \mathrm{p}<0,05)$. Le nombre de consultations est positivement corrélé avec la proportion de temps consacrée à la consultation $(\mathrm{r}=0,78, \mathrm{p}<0,01)$. La proportion de temps consacrée à la préécriture est négativement corrélée à la proportion de temps consacrée à la pause d'écriture $(\mathrm{r}=-0,44, \mathrm{p}<0,05)$. La durée de consultation est négativement corrélée à la proportion de temps consacrée à l'écriture $(r=-0,61, p<0,01)$. Le débit de production est positivement corrélé à la proportion de temps consacrée à l'écriture $(\mathrm{r}=0,56, \mathrm{p}<0,01)$.

Les deux conditions diffèrent sur deux corrélations, en lien avec la proportion de temps consacrée à la pause d'écriture. 
Tableau 2

Corrélations entre les indicateurs de temps réel pour chaque groupe d'élèves

\begin{tabular}{|c|c|c|c|c|c|c|}
\hline Classe de CE2 (8 ans) & 1. & 2. & 3. & 4. & 5. & 6. \\
\hline 1. $\mathrm{Nb}$ consultations & 1 & & & & & \\
\hline 2. Pause Pré-écriture & $-0,69^{* *}$ & 1 & & & & \\
\hline 3. Durée Consultation & $0,60^{*}$ & $-0,54 *$ & 1 & & & \\
\hline 4. Débit Production & 0,06 & $-0,11$ & $-0,42$ & 1 & & \\
\hline 5. Pause Ecriture & $-0,01$ & $-0,28$ & $-0,17$ & $-0,32$ & 1 & \\
\hline 6. Durée Ecriture & 0,08 & $-0,30$ & $-0,46$ & $0,83 * *$ & $-0,16$ & 1 \\
\hline \multicolumn{7}{|l|}{ Classe de CM1 (9 ans) } \\
\hline 1. $\mathrm{Nb}$ consultations & 1 & & & & & \\
\hline 2. Pause Pré-écriture & $-0,24$ & 1 & & & & \\
\hline 3. Durée Consultation & $0,62^{* *}$ & $-0,11$ & 1 & & & \\
\hline 4. Débit Production & $-0,10$ & 0,19 & $-0,39$ & 1 & & \\
\hline 5. Pause Ecriture & $-0,50^{*}$ & $-0,33$ & $-0,60 * *$ & $-0,26$ & 1 & \\
\hline 6. Durée Ecriture & $-0,03$ & $-0,31$ & $-0,49$ & $0,64 * *$ & $-0,10$ & 1 \\
\hline \multicolumn{7}{|l|}{ Classe de CM2 (10 ans) } \\
\hline 1. $\mathrm{Nb}$ consultations & 1 & & & & & \\
\hline 2. Pause Pré-écriture & $-0,26$ & 1 & & & & \\
\hline 3. Durée Consultation & $0,49^{*}$ & $-0,44^{*}$ & 1 & & & \\
\hline 4. Débit Production & $-0,34$ & $0,51^{*}$ & 0,03 & 1 & & \\
\hline 5. Pause Ecriture & 0,08 & $-0,55^{*}$ & $-0,17$ & $-0,63^{* *}$ & 1 & \\
\hline 6. Durée Ecriture & $-0,45$ & 0,30 & $-0,58 * *$ & 0,30 & $-0,51^{*}$ & 1 \\
\hline
\end{tabular}

Note : corrélation significative à : * $\mathrm{p}<.05 ; * * \mathrm{p}<.01$

Classe de CE2 (élèves de 8 ans). Les analyses révèlent quatre corrélations significatives. Le nombre de consultations est négativement corrélé à la pause de préécriture $(\mathrm{r}=-0,69, \mathrm{p}<$ $0,01)$; il est positivement corrélé à la durée de consultation $(\mathrm{r}=0,60, \mathrm{p}<0,05)$. La pause de préécriture est négativement corrélée à la durée de consultation $(\mathrm{r}=-0,54, \mathrm{p}<0,05)$. Et enfin, le débit de production est positivement corrélé à la durée d'écriture $(\mathrm{r}=0,83, \mathrm{p}<0,01)$.

Classe de CM1 (élèves de 9 ans). Tout comme pour les élèves de CE2, les analyses révèlent quatre corrélations significatives. Le nombre de consultation est positivement corrélé à la durée de consultation $(\mathrm{r}=0,62, \mathrm{p}<0,01)$; il est négativement corrélé aux pauses d'écriture $(\mathrm{r}=$ $0,50, \mathrm{p}<0,05)$. La durée de consultation est négativement corrélée aux pauses d'écriture $(\mathrm{r}=$ $0,60, \mathrm{p}<0,01)$. Le débit de production est positivement corrélé à la durée d'écriture $(\mathrm{r}=0,64, \mathrm{p}<$ $0,01)$.

Classe de CM2 (élèves de 10 ans). Les analyses mettent en évidence sept corrélations significatives. Le nombre de consultations est positivement corrélé à la durée de consultation $(\mathrm{r}=$ $0,49, \mathrm{p}<0,05)$. La pause de préécriture est négativement corrélée à la durée de consultations $(\mathrm{r}=$ $-0,44, \mathrm{p}<0,05)$; elle est positivement corrélée au débit de production $(\mathrm{r}=.51, \mathrm{p}<.05)$ et négativement à la pause d'écriture $(\mathrm{r}=-0,55, \mathrm{p}<0,05)$. La durée de consultation est négativement corrélée avec la durée d'écriture $(\mathrm{r}=-0,58, \mathrm{p}<0,01)$. Le débit de production est négativement corrélé à la pause d'écriture $(\mathrm{r}=-0,63, \mathrm{p}<0,01)$. Et enfin, la pause d'écriture est négativement corrélée à la durée d'écriture $(\mathrm{r}=-0,51, \mathrm{p}<0,05)$. 


\section{Discussion}

Les analyses des paramètres temporels de la production ont porté sur les indicateurs essentiels de l'activité rédactionnelle. Nos données ont montré que la proportion de temps consacrée à la pause de préécriture ne permettait de différencier ni les participants en fonction de leur groupe d'appartenance, ni les conditions de récits en fonction du nombre d'occurrences du personnage. Autrement dit, la pause de préécriture, souvent décrite comme l'activité initiale de planification conceptuelle des contenus à linéariser, occupait une proportion de temps comparable pour tous les participants, et ce, quelle que soit la condition. Ce résultat est important au sens où plusieurs travaux réalisés sur les narrations orales suggéraient que les plus jeunes participants (4-7 ans), contrairement aux plus âgés (8-9 ans), commençaient immédiatement à produire un récit, dès qu'ils découvraient les stimuli (souvent des BD), du fait d'une planification initiale faible, voire inexistante (Karmiloff-Smith, 1985 ; Hickmann et al. 1995; Vion et Colas, 1999). Nos résultats suggèrent que ce comportement serait plutôt valable à l'oral, mais pas forcément à l'écrit. Notons que dans les études antérieures, le stimulus restait constamment visible tout au long de l'activité, et les participants pouvaient s'y référer à tout moment, ce qui ne rendait pas la planification préalable nécessaire et obligatoire. Dans notre étude, l'accès au stimulus, ainsi que sa durée d'affichage, relevait de l'activité spécifique du participant et la consigne rédactionnelle insistait sur ce point. Toutefois, cette différence de conditions de présentation ne permet pas, à elle seule, d'expliquer l'absence de différence pour la pause de préécriture.

Quand nous avions examiné les comportements de consultation de la planche de BD au cours de l'activité, des différences apparaissaient entre les groupes d'élèves (nombre et durée des consultations). Ces pauses autogérées par les participants indiquaient des comportements spécifiques d'un groupe à l'autre. Comparativement aux élèves des CE2 et CM2, les élèves du CM1 consultaient plus fréquemment la planche de BD et consacraient une part importante de leur temps de production à cette activité. Autrement dit, dans leur activité d'écriture, les élèves du CM1 faisaient montre d'une gestion au coup par coup, d'un aller-retour fréquent entre la planche de BD et leur texte en cours d'élaboration. Ce comportement dénotait un empan de planification plutôt réduit. En plus des pauses de consultation, nous avions analysé les pauses d'écriture supérieures à 100ms : il s'agissait de pauses qui se produisaient au cours de l'activité d'écriture et qui ne donnaient pas lieu à une consultation de la planche de BD. Nos résultats mettaient en évidence des différences significatives entre les groupes d'élèves et des effets du nombre d'occurrences du personnage, et ceci, quel que soit le groupe. Ces pauses en cours d'écriture participent sans doute à l'organisation et à la structuration des portions du texte à produire. Des travaux antérieurs ont déjà montré que les pauses d'écriture étaient des indicateurs de traitements parallèles ou de retour sur le texte (Alamargot et al. 2007); par conséquent, elles pourraient constituer des périodes aux cours desquelles les scripteurs organisent la chaîne de référence à un personnage dans un texte (différence de corrélation d'une condition à l'autre). Enfin, l'examen de la proportion de temps consacrée à l'écriture stricto-sensu révélait également des différences entre les groupes de participants. Plus précisément, les participants du CM2 consacraient plus de temps à composer leur texte que les participants du CE2 et du CM1. L'ensemble de ces données semble indiquer que les trois groupes de participants mettaient en œuvre des stratégies différentes de gestion du temps au cours de la production.

Les analyses corrélationnelles ont montré que les pauses d'écriture n'étaient pas utilisées de la même façon, suivant les conditions de présentation ( 2 corefs vs 4 corefs). Dans la condition 1 (2 corefs), plus les participants consacraient du temps à la pause d'écriture, moins ils consacraient du temps à consulter la planche de BD. Dans la condition 2 (4 corefs) les 
participants consacraient d'autant plus de temps à la pause d'écriture qu'ils en avaient consacrée moins à la préécriture. Il est sans doute prématuré de tirer des conclusions définitives sur les fonctions de ces pauses d'écriture, mais il semble bien qu'elles assurent des rôles différents suivant les conditions de présentation. L'autre aspect important révélé par ces analyses concerne les fonctionnements différents pour chaque groupe d'élèves. Ainsi, les élèves de CE2 consacraient peu de temps à la préécriture, mais plus de temps à l'activité de consultations. Les élèves de CM1 consacraient peu de temps aux activités de consultation, mais faisaient plutôt des pauses d'écriture plus longues. Enfin, les élèves de CM2 consacraient beaucoup de temps aux activités de consultation et beaucoup de temps à l'écriture proprement dite. Autrement dit, les stratégies rédactionnelles mises en œuvre dans les différents groupes d'élèves n'étaient pas les mêmes. Ces stratégies se modifiaient progressivement en fonction du niveau de développement des élèves. Toutefois les faibles effectifs de nos groupes n'ont pas permis de préciser davantage ces stratégies en fonction des conditions de présentation.

\section{Conclusion}

L'originalité de cette recherche résidait dans l'examen de l'impact du traitement de la coréférence chez les participants de 8 à 11 ans en utilisant un dispositif de pistage de l'activité en temps réel des participants. Nous montrons que le traitement de la coréférence affecte des dimensions fines de la production écrite, notamment celles liées aux aspects fonctionnels et à la gestion des pauses en cours de production. Nos résultats contribuent à la compréhension des indicateurs qui pèsent sur le traitement de la coréférence en production écrite chez les jeunes élèves. Ils complètent les données du domaine en précisant, par exemple, les moments au cours desquels certaines opérations sont réalisées au cours de la production de texte. Toutefois, des études supplémentaires permettront sans doute de préciser davantage les modalités d'organisation et de structuration des chaînes coréférentielles au cours de l'élaboration du texte. Nous pensons en effet qu'un échantillon plus important et des conditions plus discriminantes permettraient d'apporter un nouvel éclairage sur les contraintes liées au traitement des chaînes coréférentielles en production de texte.

\section{Références}

Alamargot, D., Chesnet, D., Dansac, C., \& Ros, C. (2006). Eye and pen: A new device for studying reading during writing. Behavior Research Methods, 38(2), 287-299.

Alamargot, D., Dansac, C., Chesnet, D. \& Fayol, M. (2007). Parallel processing before and after pauses: A combined analysis of graphomotor and eye movements during procedural text production. In M. Torrance, L. v. Waes, \& D. Galbraith (Eds.), Writing and cognition: Research and applications (pp. 13-29). Amsterdam: Elsevier

Albrecht, J. E., \& Clifton, C. J. (1998). Accessing singular antecedents in conjoined phrases. Memory and Cognition, 26(3), 599-610.

Apothéloz, D. (1989). Aspects cognitifs des procédures de la cohésion textuelle. Duisburg: L.A.U.D.

Ariel, M. (1988). Referring and accessibility. Journal of linguistics, 24(01), 65-87.

Ariel, M. (1990). Accessing noun-phrase antecedents. London: Routledge.

Ariel, M. (1991). The function of accessibility in a theory of grammar. Journal of Pragmatics, 16(5), 443-463.

Arnold, J. E. (2001). The effect of thematic roles on pronoun use and frequency of reference continuation. Discourse Processes, 31, 137-162.

Arnold, J. E., \& Griffin, Z. M. (2007). The effect of additional characters on choice of referring 
expression: Everyone counts. Journal of Memory and Language, 56, 521-536.

Bartlett, E. J. (1984). Anaphoric reference in written narratives of good and poor elementary school writers. Journal of Verbal Learning and Verbal Behavior, 23(4), 540-552.

Bates, E. \& Devescovi, A. (1989). Crosslinguistic studies of sentence production. In:

MacWhinney, B., Bates, E. The Crosslinguistic Study of Sentence Processing (pp. 225253). Cambridge: Cambridge University Press

Bates, E. \& MacWhinney, B., (1989). Functionalism and the competition model. In:

MacWhinney, B., Bates, E. The Crosslinguistic Study of Sentence Processing (pp. 3-73).

Cambridge: Cambridge University Press

Bereiter, C. \& Scardamalia, M. (1987). The psychology of written composition. Hillsdale, NJ: Erlbaum

Berninger, V. W. (1999). Coordinating transcription and text generation in working memory during composing: automatic and constructive processes. Learning Disability Quarterly, 22(2), 99-112.

Berninger, V. W., Fuller, F., \& Whitaker, D. (1996). A process model of writing development across the life span. Educational Psychology Review, 8(3), 193-218.

Berninger, V. W., \& Swanson, H. L. (1994). Modifying Hayes and Flower's model of skilled writing to explain beginning and developing writing. In E. Butterfield (Ed.). Children's writing: Toward a process theory of the development of skilled writing (pp. 57-81). Greenwich, CT: JAI Press.

Berninger, V. W., Vaughan, K. B., Abbott, R. D., Abbott, S. P., Rogan, L. W., Brooks, A., Reed, E. \& Graham, S. (1997). Treatment of handwriting problems in beginning writers: Transfer from handwriting to composition. Journal of Educational Psychology, 89(4), 652-666.

Botvin, G. J., \& Sutton-Smith, B. (1977). The development of structural complexity in children's fantasy narratives. Developmental Psychology,13(4), 377-388.

Bourdin, B., Leuwers, C. \& Bourdon, C. (2011). Impact des contraintes linguistiques et cognitives sur l'acquisition de l'accord en genre de l'adjectif en français écrit. Psychologie Française, 56(3), 133-143.

Bouchard, M.-E. G., Fitzpatrick, E. M. \& Olds, J. (2009. Analyse psychométrique d'outils d'évaluation utilizes auprès des enfants francophones. Revue canadienne d'orthophonie et d'audiologie, 33(3), 129-139.

Brennan, S. E. (1995). Centering attention in discourse. Language and Cognitive Processes, 10(2), 137-167.

Brennan, S. E., \& Clark, H. H. (1996). Conceptual pacts and lexical choice in conversation. Journal of Experimental Psychology: Learning, Memory, and Cognition, 22, 1482-1493.

Camblin, C. C., Ledoux, K., Boudewyn, M. Godon, P. C., \& Swaab, T. Y. (2007). Processing new and repeated names: Effects of coreference on repetition priming with speech and fast RSVP. Brain Research, 1146, 172-184.

Chanquoy, L. (2001). How to make it easier for children to revise their writing: A study of text revision from 3rd to 5th grades. British Journal of Educational Psychology, 71(1), 15-41.

Chanquoy, L., \& Alamargot, D. (2002). Mémoire de travail et rédaction de textes: évolution des modèles et bilan des premiers travaux. L'Année Psychologique, 102(2), 363-398.

Charolles, M. (1988). Les plans d'organisation textuelle: périodes, chaînes, portées et séquences. Pratiques, 57, 3-13. 
Chesnet, D. \& Alamargot, D. (2005). Analyse en temps réel des activités oculaires et graphomotrices du scripteur : intérêt du dispositif «Eye and Pen ». L'Année Psychologique, 105(3), 477-520.

Clancy, P.M. (1980). Referential choice in English and Japanese. In: W.L. Chafe (Ed.), The pear stories: Cognitive, cultural, and linguistic aspects of narrative production (127-199). Norwood, New Jersey: Ablex.

Cohen, J. (1988). Statistical power analysis for the behavioral sciences (2nd ed.). Hillsdale, NJ: Erlbaum

Corblin, F. (1995). Les formes de reprise dans le discours. Anaphores et chaînes de réféerence. Rennes : PUR.

Dedeyan, A., Largy, P. \& Negro, I. (2006). Mémoire de travail et détection d'erreurs d'accord verbal : étude chez le novice et l'expert. Langage, 40(164), 57-70.

Doutriaux, F., \& Lepez, R. (1980). TNO : Test de niveau d'orthographe. Paris: ECPA

Ehrlich, K. \& Rayner, K. (1983). Pronoun assignment and semantic integration during reading: Eye movements and immediacy of processing. Journal of Verbal Learning and Verbal Behavior, 22(1), 75-87.

Espéret, E., Chesnet, D., Ploquin, N. \& Crété, M.-F. (1995). Processus cognitifs mis en jeu dans la production écrite : sont-ils modifiés par le traitement de texte ? Repères, 1995.

Favart, M. \& Olive, T. (2005). Modèles et méthodes d'étude de la production écrite. Psychologie française, 50(3), 273-285.

Fayol, M. (1986). Les connecteurs dans les récits écrits: étude chez l'enfant de 6 à 10 ans. Pratiques: théorie, pratique, pédagogie, (49), 101-113.

Fayol, M., Foulin, J. -N., \& Chanquoy, L. (1989). Approche en temps réel de la production des connecteurs et de la ponctuation : vers un modèle procédural de la composition écrite. Langue Française, 81(1), 5-20.

Ferreira, V. S., Slevc, L. R., \& Rogers, E. S. (2005). How do speakers avoid ambiguous linguistic expressions? Cognition, 96, 263-284.

Ferreiro, E. (1988). L'écriture avant la lettre. In H. Sinclair, (Eds), La production de notations chez le jeune enfant (pp. 17-70). Paris : PUF.

Fijalkow, J. (1993). Entrée dans l'écrit. Paris : Magnard.

Foulin, J.-N. (1995). Pauses et débits : les indicateurs temporels de la production écrite. L'année Psychologique, 95(3), 483-504.

Foulin, J.-N. \& Fayol, M. (1988). Etude en temps réel de la production de texte chez les enfants de sept et huit ans. European Journal of Psychology of Education, 3(4), 461-475.

Fukumura, K., \& van Gompel, R. (2010). Choosing anaphoric expressions: Do people take into account likelihood of reference? Journal of Memory and Language, 62(1), 52-66.

Fukumura, K., van Gompel, R. P. G., Harley, T. \& Pickering, M. (2011). How does similaritybased interference affect the choice of referring expression? Journal of Memory and Language, 65, 331-344.

Garrod, S., Freudenthal, D., \& Boyle, E. (1994). The role of different types of anaphors in the online resolution of sentences in a discourse. Journal of Memory and Language, 33(1), 3968.

Gernsbacher, M. A. (1989). Mechanisms that improve referential access. Cognition, 32(2), 99156.

Givón, T. (1983). Topic continuity in discourse: An introduction. In T. Givón (Ed.), Topic continuity in discourse: A quantitative cross-language study (pp. 1-41). Amsterdam: John Benjamins. 
Gordon, P. C., Grosz, B. J., \& Gilliom, L. A. (1993). Pronouns, names, and the centering of attention in discourse. Cognitive science, 17(3), 311-347.

Grosz, B. J., Joshi, A. K., \& Weinstein, S. (1995). Centering: A framework for modeling the local coherence of discourse. Computational Linguistics, 21(2), 203-225.

Hayes, J. R., \& Flower, L. (1980). Identifying the organization of writing processes. In Gregg, W. \& Steinberg, E.R. (Eds.), Cognitive Processes in Writing (pp. 3-30). Hillsdale, NJ: Erlbaum.

Hickmann, M., Kail, M., \& Roland, F. (1995). Cohesive anaphoric relations in French children's narratives as a function of mutual knowledge. First language, 15, 277-300.

Howell, D. C. (1998). Méthodes statistiques en sciences humaines. Bruxelles : De Boeck.

Hickmann, M., \& Schneider, P. (1993). Children's ability to restore the referential cohesion of stories. First Language, 13(38), 169-202.

Kail, M., \& Hickmann, M. (1992). French children's ability to introduce referents in narratives as a function of mutual knowledge. First language, 12(34), 73-94.

Karmiloff-Smith, A. (1985). Language and cognitive processes from a developmental perspective. Language and cognitive processes, 1(1), 61-85.

Karmiloff-Smith, A. (1992). Beyond the Modularity: A developmental perspective on cognitive science. London: The MIT Press.

Kellogg, R. T. (1996). A model of working memory in writing. In M. C. Levy, \& S. E. Ransdell (Eds.), The science of writing: Theories, methods, individual differences and applications (pp. 57-71). Hillsdale, NJ: Laurence Erlbaum Associates.

Laudec et Cauvin, R. (1997). Cedric : chaud et froid. Tome 6. Bruxelles: Dupuis.

Ledoux, K., Gordon, P. C., Camblin, C. C., \& Swaab, T. Y. (2007). Coreference and lexical repetition: Mechanisms of discourse integration. Memory \& cognition, 35(4), 801-815.

Matsuhashi, A. (1981). Pausing and planning: The tempo of written discourse production. Research in The Teaching of English, 15, 113-134.

McCutchen, D. (1996). A capacity theory of writing: Working memory in composition. Educational Psychology Review, 8(3), 299-325.

McKeough, A., \& Genereux, R. (2003). Transformation in narrative thought during adolescence: The structure and content of story compositions. Journal of Educational Psychology, 95(3), 537-552.

Millogo, V. E. (2005). The use of anaphoric pronouns by French children in narrative: Evidence from constrained text production. Journal of child language, 32(2), 439-461.

Morin, M.-F., \& Montésinos-Gelet, I. (2007). Effet d'un programme d'orthographes approchées en maternelle sur les performances ultérieures en lecture et en écriture d'élèves à risque. Revue des Sciences de l'Education, 33(3), 663-683.

Murphy, G. L. (1985). Processes of understanding anaphora. Journal of Memory and Language, 24, 290-303.

Murphy, K. R., \& Myors, B. (2004). Statistical Power Analysis: A Simple and General Model for Traditional and Modern Hypothesis Tests. $2^{\text {nd }}$ ed. Mahwah, NJ: Erlbaum

Nicol, J., \& Swinney, D. (1989). The role of structure in coreference assignment during sentence comprehension. Journal of psycholinguistic research, 18(1), 5-19.

O'Brien, E. J., Raney, G. E., Albrecht, J. E., \& Rayner, K. (1997). Processes involved in the resolution of explicit anaphors. Discourse Processes, 23(1), 1-24.

Olive, T. (2002). La gestion en temps réel de la production verbale: méthodes et données. In M. Fayol (Ed). Production du langage (pp. 131-147). Paris: Hermès Science Publications. 
Olive, T., \& Piolat, A. (2005). Le rôle de la mémoire de travail dans la production écrite de textes. Psychologie française, 50(3), 373-390.

Piolat, A. (2004). Approche cognitive de l'activité rédactionnelle et de son acquisition. Le rôle de la mémoire de travail. Linx. Revue des linguistes de l'université Paris X Nanterre, (51), $55-74$.

Rathmann, C., Mann, W. \& Morgan, G. (2007). Narrative structure and narrative development in Deaf Children. Deafness and Education International 9(4): 187-196.

Reichler-Béguelin, M. J. (1988). Anaphore, cataphore et mémoire discursive. Pratiques, 57, 1543.

Richardson, J. T. (2011). Eta squared and partial eta squared as measures of effect size in educational research. Educational Research Review, 6(2), 135-147.

Shiro, M. (2003). Genre and evaluation in narrative development. Journal of Child Language, 30, 165-195.

Stadler, M. A., \& Ward, G. C. (2005). Supporting the narrative development of young children. Early Childhood Education Journal, 33(2), 73-80.

Stevenson, R. J., Crawley, R. A., \& Kleinman, D. (1994). Thematic roles, focus and the representation of events. Language and Cognitive Processes, 9(4), 519-548.

Van Galen, G. P. (1990). Phonological and motoric demands in handwriting: Evidence for discrete transmission of information. Acta Psychologica, 74(2), 259-275.

Vion, M., \& Colas, A. (1999). Expressing coreference in French: cognitive constraints and development of narrative skills. Journal of Psycholinguistic Research, 28 (3), 261-291.

Vion, M., Piolat, A., \& Colas, A. (1989). Oral and written reference to new and given information by 9 and 11 year-olds and adults. European Journal of Psychology of Education, 4, 37-49. 
ANNEXE 1 : Planche de BD avec les deux conditions de présentation

\section{Condition 1}

Vignette 1

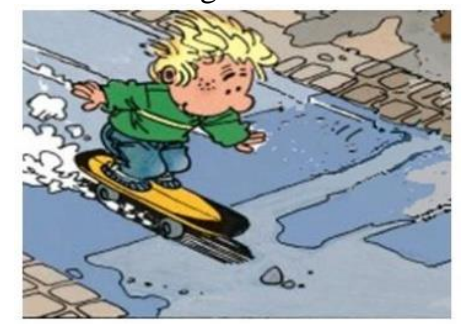

Vignette 4

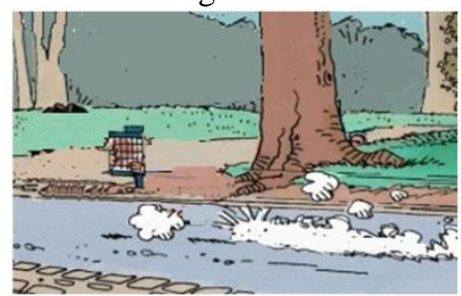

Condition 2

Vignette 1

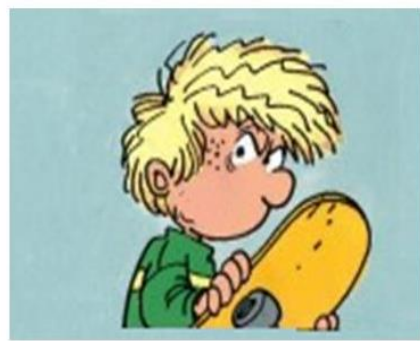

Vignette 4

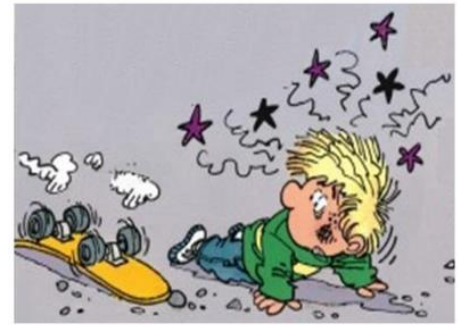

Vignette 2

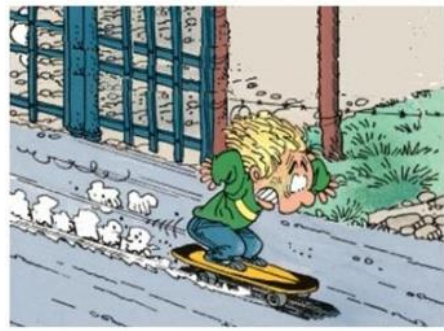

Vignette5

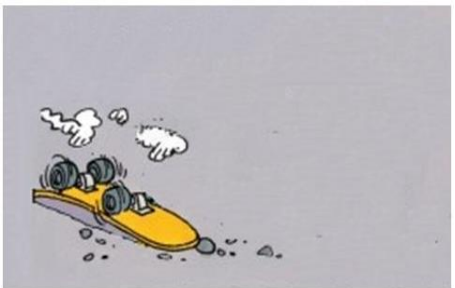

Vignette 2

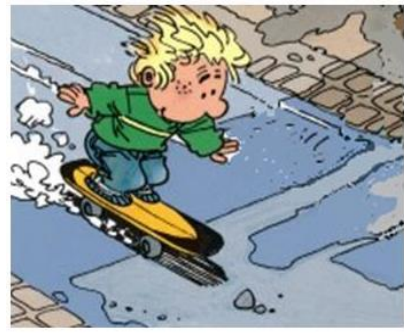

Vignette 5

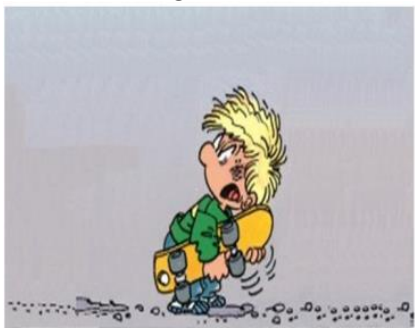

Vignette 3

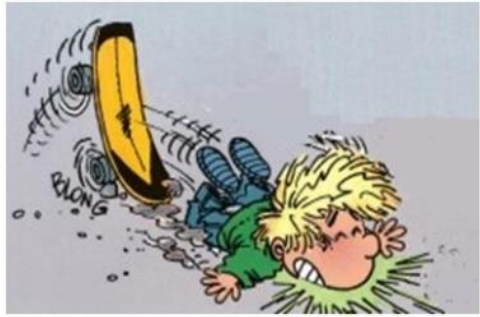

Vignette 3

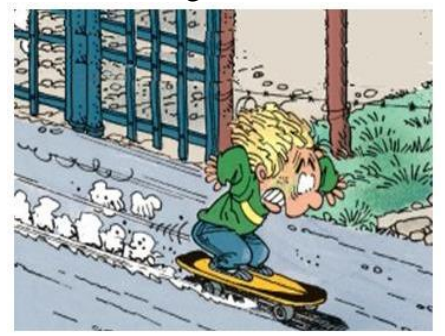

\section{Bios auteur}

Victor Millogo est enseignant-chercheur à l'Université de Poitiers, à l'Ecole Supérieure du Professorat et de l'Education (ESPE de l'Académie de Poitiers), France. Il conduit ses recherches au Centre de Recherche sur la Cognition et l'Apprentissage (CeRCA UMR CNRS 7295) de Poitiers. 